\title{
Fast Adaptive S-ALOHA Scheme for Event-driven Machine-to-Machine Communications
}

\author{
Huasen $\mathrm{Wu}^{* \dagger}$, Chenxi Zhu ${ }^{\ddagger}$, Richard J. La ${ }^{\S}$, Xin Liu ${ }^{\dagger}$, and Youguang Zhang* \\ ${ }^{*}$ School of Electronic and Information Engineering, Beihang University, Beijing 100191, China \\ Email: huasenwu@gmail.com \\ $\dagger$ Department of Computer Science, University of California, Davis, CA 95616, USA \\ $\ddagger$ Mallard Creek Networks, 11452 Mallard Creek Trail, Fairfax, VA 22033, USA \\ $\S$ Department of Electrical and Computer Engineering, University of Maryland, College Park, MD 20742, USA
}

\begin{abstract}
Machine-to-Machine (M2M) communication is now playing a market-changing role in a wide range of business world. However, in event-driven M2M communications, a large number of devices activate within a short period of time, which in turn causes high radio congestions and severe access delay. To address this issue, we propose a Fast Adaptive S-ALOHA (FASA) scheme for M2M communication systems with bursty traffic. The statistics of consecutive idle and collision slots, rather than the observation in a single slot, are used in FASA to accelerate the tracking process of network status. Furthermore, the fast convergence property of FASA is guaranteed by using drift analysis. Simulation results demonstrate that the proposed FASA scheme achieves near-optimal performance in reducing access delay, which outperforms that of traditional additive schemes such as PB-ALOHA. Moreover, compared to multiplicative schemes, FASA shows its robustness even under heavy traffic load in addition to better delay performance.
\end{abstract}

\section{INTRODUCTION}

Machine-to-Machine (M2M) communication or MachineType Communication (MTC) is expected to be one of the major driving forces of cellular networks, as its demand is increasing greatly in recent years $[1,2]$. Behind the proliferation of $\mathrm{M} 2 \mathrm{M}$ communication, the congestion problems in M2M communication become a big concern. The reason is that the device density of M2M communication is much higher than that in traditional Human-to-Human $(\mathrm{H} 2 \mathrm{H})$ communication [1]. What's worse, in event-driven M2M applications, many devices may be triggered almost simultaneously and attempt to access the base station (BS) through the Random Access Channel (RACH) [3]. Such high burstiness can result in congestion and increase response time, which motivates our research.

In literature, several strategies have been proposed for avoiding radio congestions in M2M communication [4-8]. Among these strategies, Slotted-ALOHA (S-ALOHA) type policies, e.g., access class barring (ACB) based schemes in $[6,7]$, are applied in 3GPP for random access control of M2M devices. In these schemes, it is left to users to decide the operation parameters such as transmission probability to stabilize and optimize the system. Two typical classes of schemes, additive and multiplicative schemes, have been proposed for stabilizing the S-ALOHA system. Historical outcomes are applied in these schemes to estimate the network status and optimize the access probability. However, as discussed in more detail later, traditional additive schemes, such as Pseudo Bayesian ALOHA (PB-ALOHA) [9], estimate the number of backlogged devices based on the observation of the previous slot and cannot adjust the transmission probability in time under highly busty traffic, which results in large access delay. The delay can be shortened in multiplicative schemes [10], e.g., Q-Algorithm [11] and its enhanced version $\mathrm{Q}^{+}$-Algorithm [12]. The reason is that the estimate has exponential increment in consecutive collision slots and exponential decrement in idle slots, which means multiplicative schemes can track the network status in a short period. However, the throughput suffers in these schemes due to the fluctuations in the estimation [10]. More recently, Adaptive Traffic Load Slotted Multiple Access Collision Avoidance (ATL S-MACA) mechanism in [8] uses packet sensing and adaptive method to improve the access performance under heavy traffic load. But the scheme is designed for M2M communications with Poisson traffic and is not suitable for eventdriven M2M applications.

In this paper, we propose a Fast Adaptive S-ALOHA (FASA) scheme for access control of event-driven M2M communications. In order to deal with congestions resulted from the burstiness, we collect access results in the past slots, in particular, consecutive idles or collisions, and apply them to track the network status. Furthermore, using drift analysis, we carefully design the parameters in the scheme such that the transmission probability can converge quickly to the optimal value. With numerical simulations, we show that the proposed FASA scheme can achieve the near-optimal performance in reducing the access delay, as well as robust performance under all traffic loads less than $e^{-1}$, which is the maximum throughput of $\mathrm{S}$ ALOHA system.

The remainder of the paper is organized as follows. In Section II, we present the system model, including the bursty traffic model for event-driven M2M communications. In Section III, after analyzing the limit of traditional fixed step-size adaptive policies, we propose the FASA scheme and design it based on drift analysis. In Section IV, simulation results are presented to evaluate the performance of the proposed scheme, compared with the theoretical optimal scheme, PB-ALOHA, and $\mathrm{Q}^{+}$Algorithm. In Section V, we discuss future work and conclude the paper.

\section{System MODEL}

In this paper, we consider S-ALOHA based random access control for bursty M2M communication traffic. The system consists of a BS and a large number of M2M devices, where devices with data attempt to access the BS through a single $\mathrm{RACH}$. The time is divided into time slots, each of which is long enough to transmit a request packet. Deferred first transmission (DFT) mode [9] is assumed, in which a device with a new request packet immediately goes to backlogged state. In slot $t$, all backlogged devices transmit packets with 
probability $p_{t}$, which is broadcasted by the BS at the beginning of the slot. Moreover, an ideal collision channel is assumed, where the transmitted packet will be successfully received by the BS when no other packets are being transmitted in the same slot. Let $Z_{t}$ denote the access result in slot $t$, and $Z_{t}=0,1$, or $c$ depending on whether zero, one, or more than one request packets are transmitted on $\mathrm{RACH}$. At the end of slot $t$, the BS decides the transmission probability for next slot based on the sequence $\left\{Z_{0}, Z_{1}, \ldots, Z_{t}\right\}$, i.e.,

$$
p_{t+1}=\Pi_{t}\left(Z_{0}, Z_{1}, \ldots, Z_{t}\right) .
$$

The objective of the BS is to maximize the throughput and minimize the access delay. It is well known that, when $N_{t} \geq 1$ in slot $t$, where $N_{t}$ is the number of backlogged devices in slot $t$, using transmission probability $p_{t}=1 / N_{t}$ maximizes the throughput of the S-ALOHA system. However, the BS does not know $N_{t}$ and has to obtain its estimate $\hat{N}_{t}$ based on the access results in the past.

For the traffic model, we focus on event-driven M2M applications, i.e., when an event is detected, a random and large number of M2M devices become active almost simultaneously and attempt to access the BS on the RACH. We call this an active stage. To capture the burstiness of M2M traffic, instead of assuming Poisson arrival process, we assume that when an event is detected, $N$ devices are triggered in a short duration $T$. As suggested in [6], the active time follows the beta distribution, of which the probability density function is

$$
f(x)=\frac{x^{\alpha-1}(T-x)^{\beta-1}}{T^{\alpha+\beta-1} B(\alpha, \beta)}, x \in[0, T],
$$

where $B(\alpha, \beta)$ is the beta function, and [6] suggests that the values $\alpha=3$ and $\beta=4$ give the best fit. The number of active devices $N$ and the active duration $T$ are both random variables and no prior knowledge about them is assumed at the BS. For the sake of simplicity, a backlogged device will not generate any new requests since the new coming data can be transmitted as long as the device accesses the BS successfully. On the other hand, all the backlogged devices will keep retransmitting until their request packets are successfully received by the BS.

\section{FAST ADAPTIVE S-ALOHA}

The estimation of the number of backlogged devices plays an important part in stabilizing and optimizing the S-ALOHA system. In this section, using drift analysis, we first examine the limit of traditional fixed step-size estimation schemes. Then, we propose and analyze a fast adaptive scheme, referred to as Fast Adaptive S-ALOHA.

\section{A. Drift Analysis of Fixed Step-size Estimation Schemes}

Many additive schemes with fixed step-size have been proposed to estimate the number of backlogged devices. A unified framework of these schemes is proposed and studied by Kelly in [13], where the estimate $\hat{N}_{t}$ is updated by the recursion

$\hat{N}_{t+1}=\max \left\{1, \hat{N}_{t}+a_{0} I\left[Z_{t}=0\right]+a_{1} I\left[Z_{t}=1\right]+a_{c} I\left[Z_{t}=c\right]\right\}$,

where $a_{0}, a_{1}$, and $a_{c}$ are constants and $I[A]$ is the indicator function of event $A$.

With the estimation, the BS sets the transmission probability $p_{t}=1 / \hat{N}_{t}$ for all backlogged devices, and thus the offered load $\rho=N_{t} p_{t}=N_{t} / \hat{N}_{t}$, representing the average number of devices attempting to access the channel. To stabilize and optimize the S-ALOHA system, $\hat{N}_{t}$ needs to track the actual number of backlogged devices $N_{t}$, especially when $N_{t}$ is large. When $N_{t}=n$ and $\hat{N}_{t}=\hat{n}$, the estimation drift can be calculated as [13]

$$
\begin{aligned}
& E\left[\hat{N}_{t+1}-\hat{N}_{t} \mid N_{t}=n, \hat{N}_{t}=\hat{n}\right] \\
= & \left(a_{0}-a_{c}\right)\left(1-\frac{1}{\hat{n}}\right)^{n}+\left(a_{1}-a_{c}\right) \frac{n}{\hat{n}}\left(1-\frac{1}{\hat{n}}\right)^{n-1}+a_{c} \\
\rightarrow & \left(a_{0}-a_{c}\right) e^{-\rho}+\left(a_{1}-a_{c}\right) \rho e^{-\rho}+a_{c} \stackrel{\text { def }}{=} \Delta(\rho),
\end{aligned}
$$

as $n \rightarrow \infty$, with $n / \hat{n}=\rho$ fixed.

By properly choosing the parameters $a_{i}(i=0,1, c)$ such that $\Delta(\rho)<0$ if $\rho<1$ and $\Delta(\rho)>0$ if $\rho>1$, the estimate $\hat{N}_{t}$ will drift towards the true value and thus the $\mathrm{S}$ ALOHA system can be stabilized. However, these fixed stepsize schemes are not suitable for systems with bursty traffic. When the estimate $\hat{N}_{t}$ deviates far away from the true value $N_{t}$, we have $\lim _{\rho \rightarrow 0} \Delta(\rho)=a_{0}$ and $\lim _{\rho \rightarrow \infty} \Delta(\rho)=a_{c}$. These limits indicate that the drift tends to be a constant even when the deviation is large, which could result in a large tracking time. Thus, it is necessary to design fast estimation schemes for event-driven M2M communication.

\section{B. Design of FASA}

As analyzed in the previous subsection, fixed step-size estimation schemes such as PB-ALOHA may not be sufficient to adapt in a timely manner for systems with bursty traffic because it uses a constant step-size even when the estimate is far away from the true value. We note that in addition to the access result in the previous slot, the access results in several consecutive slots will be helpful for improving the estimation as they may reveal additional information about the true value. Intuitively, collisions in several consecutive slots are likely caused by a significant underestimation, i.e., $\hat{N}_{t} \ll N_{t}$, and the BS should aggressively increase its estimate. Similarly, several consecutive idle slots may indicate that the estimate $N_{t} \gg N_{t}$, and it should be reduced aggressively.

Motivated by this intuition, we propose a FASA scheme that updates $\hat{N}_{t}$ as follows

$$
\hat{N}_{t+1}= \begin{cases}\max \left\{1, \hat{N}_{t}-1-h_{0}(\nu)\left(K_{0, t}\right)^{\nu}\right\}, & \text { if } Z_{t}=0 \\ \hat{N}_{t}, & \text { if } Z_{t}=1 \\ \hat{N}_{t}+\frac{1}{e-2}+h_{c}(\nu)\left(K_{c, t}\right)^{\nu}, & \text { if } Z_{t}=c\end{cases}
$$

where $K_{0, t}$ and $K_{c, t}$ are the numbers of consecutive idle and collision slots up to slot $t$, respectively; $\nu>0$ is the parameter that controls the adjusting speed; $h_{0}(\nu)$ and $h_{c}(\nu)$ are functions of $\nu$ that guarantee the tracking criterions [13]. We are mostly interested in those cases where the number of backlogged devices is large. Hence we will approximate $\max \{1, x\}$ as $x$ for notational simplicity in the analysis later.

Next, we design $h_{0}(\nu)$ and $h_{c}(\nu)$ by drift analysis. Consider tracking a fixed number of backlogged devices, i.e., $N_{t}=n$ is constant for all $t \in \mathbb{Z}_{+}:=\{0,1,2, \ldots\}$. Assume that in slot $t$, the estimate $\hat{N}_{t}=\hat{n}$, and thus the offered load $\rho=n / \hat{n}$. When $n$ is large, the drift of estimation for FASA can be calculated as

$$
\begin{aligned}
& \Delta_{F A S A}(\rho)=E\left[\hat{N}_{t+1}-\hat{N}_{t}\right] \\
= & q_{0}(\rho) E\left[\Delta_{0}(\rho)\right]+q_{1}(\rho) E\left[\Delta_{1}(\rho)\right]+q_{c}(\rho) E\left[\Delta_{c}(\rho)\right],
\end{aligned}
$$


where $q_{0}(\rho)=e^{-\rho}, q_{1}(\rho)=\rho e^{-\rho}$, and $q_{c}(\rho)=1-q_{0}(\rho)-$ $q_{1}(\rho)$ are the probabilities of an idle, success, and collision slot, respectively; $\Delta_{i}(\rho)(i=0,1, c)$ is the change in $\hat{N}_{t}$ resulting from the corresponding update.

Obviously, $\Delta_{1}(\rho)=0$ since the estimated number remains unchanged when a packet is successfully transmitted in a slot. However, $\Delta_{0}(\rho)$ and $\Delta_{c}(\rho)$ depend on the distribution of $K_{0, t}$ and $K_{c, t}$, respectively. For example,

$$
E\left[\Delta_{0}(\rho)\right]=\sum_{k_{0}=1}^{t+1} E\left[\Delta_{0}(\rho) \mid K_{0, t}=k_{0}\right] \operatorname{Pr}\left(K_{0, t}=k_{0}\right) .
$$

Unfortunately, it is difficult to obtain the distribution of $K_{0, t}$ and $K_{c, t}$ directly, and thus we resort to approximation in order to make the problem tractable. It is easy to see that the update of $\hat{N}_{t}$ is mostly affected by the access results in the past $s$ slots $\left\{Z_{t-s}, Z_{t-s+1}, \ldots, Z_{t-1}\right\}$, where $s$ is the number of consecutive idles or collisions immediately proceeding $t$, or $s=1$ if $Z_{t-1}=1$. The update step is reset whenever $Z_{t-1} \neq Z_{t}$ or $Z_{t}=1$. For tractability, we approximate the estimates $\hat{N}_{t^{\prime}}\left(t^{\prime}=0,1, \ldots, t-1\right)$ as $\hat{N}_{t}$. Thus, the access outcomes $\left\{Z_{0}, Z_{1}, \ldots, Z_{t}\right\}$ are independent and identically distributed (i.i.d.) random variables, of which $q_{0}(\rho), q_{1}(\rho)$, and $q_{c}(\rho)$ are the probabilities of the idle, success, and collision slot, respectively. As we will see later, this provides a rough bound for the drift and guarantees the convergence of the algorithm.

First, to calculate the expected drift in an idle slot, suppose that no packet is transmitted in slot $t$. Then the estimated number will be reduced by $1+h_{0}(\nu)\left(K_{0, t}\right)^{\nu} . K_{0, t}=k_{0}$ $\left(k_{0} \leq t\right)$ holds when slots $t-k_{0}+1, t-k_{0}+2, \ldots, t-1$ are all idle while slot $t-k_{0}$ is not. Under the approximation that the access results are i.i.d. in each slot, and the probability that an idle slot occurs is $q_{0}(\rho)$, we have

$$
\operatorname{Pr}\left(K_{0, t}=k_{0}\right)= \begin{cases}q_{0}^{k_{0}-1}(\rho)\left[1-q_{0}(\rho)\right], & \text { if } 1 \leq k_{0} \leq t \\ q_{0}^{k_{0}-1}(\rho), & \text { if } k_{0}=t+1\end{cases}
$$

As $t$ tends to infinity, $q_{0}^{t}(\rho)$ tends to 0 , and $K_{0, t}$ can be approximated by a geometrically distributed random variable with success probability $1-q_{0}(\rho)$. Hence,

$$
\begin{aligned}
E\left[\Delta_{0}(\rho)\right] & \approx \sum_{k_{0}=1}^{\infty}\left[-1-h_{0}(\nu) k_{0}^{\nu}\right] q_{0}^{k_{0}-1}(\rho)\left[1-q_{0}(\rho)\right] \\
& =-\left[1+h_{0}(\nu) M\left(\nu, q_{0}(\rho)\right)\right]
\end{aligned}
$$

where $M\left(\nu, q_{0}(\rho)\right)$ is the approximate expectation of $\left(K_{0, t}\right)^{\nu}$ when the offered load is $\rho$, which is given by

$$
M\left(\nu, q_{0}(\rho)\right)=\sum_{k_{0}=1}^{\infty} k_{0}^{\nu} q_{0}^{k_{0}-1}(\rho)\left[1-q_{0}(\rho)\right] .
$$

Secondly, we can calculate the drift of the estimation in a collision slot in a similar fashion as follows:

$$
E\left[\Delta_{c}(\rho)\right] \approx(e-2)^{-1}+h_{c}(\nu) M\left(\nu, q_{c}(\rho)\right) .
$$

From (8) and (9), the drift of FASA can be approximated using

$$
\begin{gathered}
\Delta_{F A S A}(\rho) \approx-q_{0}(\rho)\left[1+h_{0}(\nu) M\left(\nu, q_{0}(\rho)\right)\right] \\
\quad+q_{c}(\rho)\left[(e-2)^{-1}+h_{c}(\nu) M\left(\nu, q_{c}(\rho)\right)\right] .
\end{gathered}
$$

In order to keep the estimated number $\hat{N}_{t}$ close to the true value, it is required that $\Delta_{F A S A}(\rho)=0$ for $\rho=1$. Letting $q_{0}^{*}=q_{0}(1)=e^{-1}$ and $q_{c}^{*}=q_{c}(1)=1-2 e^{-1}$, we expect that

$$
\Delta_{F A S A}(1)=-h_{0}(\nu) q_{0}^{*} M\left(\nu, q_{0}^{*}\right)+h_{c}(\nu) q_{c}^{*} M\left(\nu, q_{c}^{*}\right)=0 .
$$

Therefore, in order to satisfy the condition in (11), we can select the following $h_{0}(\nu)$ and $h_{c}(\nu)$ :

$$
\begin{aligned}
& h_{0}(\nu)=\eta\left[q_{0}^{*} M\left(\nu, q_{0}^{*}\right)\right]^{-1}, \\
& h_{c}(\nu)=\eta\left[q_{c}^{*} M\left(\nu, q_{c}^{*}\right)\right]^{-1},
\end{aligned}
$$

where $\eta>0$ is a constant and is another parameter for controlling the tracking speed.

We now interpret the effect of i.i.d. approximation. We focus on the cases of $\rho \ll 1$ and $\rho \gg 1$, since the evolution of $\hat{N}_{t}$ in these cases diverges the most from the i.i.d. approximation. If $\rho=n / \hat{n} \ll 1$ in slot $t$, it is more likely that there were consecutive idle slots and the estimate $\hat{N}_{t}$ was decreasing in the past slots, suggesting $\hat{N}_{t^{\prime}}>\hat{N}_{t}$ for slot $t^{\prime}<t$ with high probability. In this case, the idle probabilities in the past slots would have been larger than that in slot $t$, while the collision probabilities would have been smaller than that in slot $t$. Hence, using $\hat{N}_{t}$ as an approximation of $\hat{N}_{t^{\prime}}\left(t^{\prime}<t\right)$ overestimates $E\left[\Delta_{0}(\rho)\right]$ and $E\left[\Delta_{c}(\rho)\right]$, and the drift analysis above gives an upper bound of the drift for $\rho \ll 1$. Similarly, when $\rho \gg 1$, the drift analysis above gives a lower bound of the drift. Subsequently, the above analysis using the i.i.d. approximation roughly bounds the evolution of $\hat{N}_{t}$ in both directions and guarantees the convergence of $\hat{N}_{t}$ to $n$ following Proposition 1 (Section III-C).

\section{Drift Analysis of FASA}

The chosen $h_{0}(\nu)$ and $h_{c}(\nu)$ guarantee that $\Delta_{F A S A}(1)=0$ and thus provide a necessary condition for FASA to track the number of backlogged devices. Furthermore, Proposition 1, which can be proved by examining the derivative of $\Delta_{F A S A}(\rho)$, shows a desirable property of FASA. With this property, the BS inclines to decrease $\hat{N}_{t}$ when $\hat{N}_{t}>N_{t}$ and increase $\hat{N}_{t}$ when $\hat{N}_{t}<N_{t}$. Thus, $\hat{N}_{t}$ is able to track the number of backlogged devices $n$.

Proposition 1: Given that $h_{0}(\nu)$ and $h_{c}(\nu)$ are defined in (12) and (13), respectively, the approximate drift of FASA $\Delta_{F A S A}(\rho)$ is a strictly increasing function of $\rho$. In addition, $\Delta_{F A S A}(\rho)<0$ when $0<\rho<1$ and $\Delta_{F A S A}(\rho)>0$ when $\rho>1$.

Proof: See Appendix.

In order to understand better the behavior of the scheme, we now present the drift of estimation for $\nu=1,2$ and 3 . When $\nu \in \mathbb{Z}_{+}, M\left(\nu, q_{i}(\rho)\right)(i=0, c)$ is the $\nu$ th-moment of a geometrically distributed random variable with success probability $1-q_{i}(\rho)$, and its closed-form expression can be obtained. Consequently, with the expressions of $M\left(\nu, q_{i}(\rho)\right)$, we can obtain functions $h_{0}(\nu), h_{c}(\nu)$, and the drift $\Delta_{F A S A}(\rho)$ for $\nu=1,2$ and 3. For example, when $\nu=2$, the drift of FASA can be expressed as

$$
\begin{aligned}
\Delta_{F A S A}^{(2)}(\rho) & =\Delta^{(0)}+\eta\left[-\frac{(e-1)^{2}\left(e^{\rho}+1\right)}{(e+1)\left(e^{\rho}-1\right)^{2}}\right. \\
& \left.+\frac{2\left(e^{\rho}-\rho-1\right)\left(2 e^{\rho}-\rho-1\right)}{(e-2)(e-1)(\rho+1)^{2}}\right],
\end{aligned}
$$


where $\Delta^{(0)}=-e^{-\rho}+\frac{1-2 e^{-\rho}}{e-2}$ is the drift of the fixed step-size estimation (3) with $a_{0}=-1, a_{1}=0$ and $a_{c}=(e-2)^{-1}$.

As shown in Fig. 1, when the estimated number deviates far away from the actual number of backlogged devices, FASA adjusts its step-size accordingly, while PB-ALOHA still uses the same step-size. Therefore, using FASA results in much shorter adjusting time than PB-ALOHA, and thus improves the performance of $\mathrm{M} 2 \mathrm{M}$ communication systems with bursty traffic. Note that the drifts of multiplicative schemes such as $Q^{+}$-Algorithm are not illustrated here since they depend on not only the offered load $\rho$ but also the estimate $\hat{N}_{t}$.

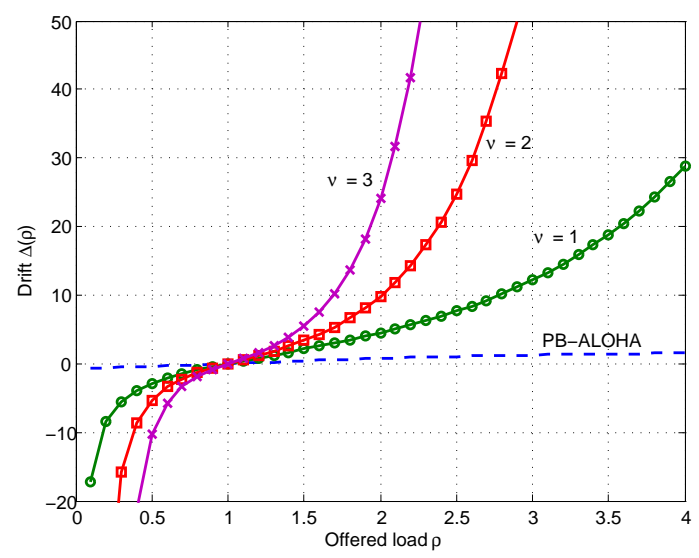

Fig. 1. Drift of estimation $(\eta=1)$

\section{Simulation Results}

In this section we evaluate the performance of the proposed scheme through simulation. We compare the performance of our FASA scheme, the ideal policy with perfect knowledge of backlog, PB-ALOHA [9], and $\mathrm{Q}^{+}$-Algorithm [12].

\section{A. Settings}

First, we choose the parameter values for the adaptive schemes. Since simulations show that the performances of FASA with different $\eta$ and $\nu$ are close, we just present the results for $\eta=1$ and $\nu=2$ in this paper due to the space limitation. With perfect knowledge of $N_{t}$, the ideal policy sets transmission probability at $p_{t}=1 / N_{t}$ for $N_{t}>0$. Thus, the ideal policy achieves the minimum access delay of S-ALOHA and serves as a benchmark in the comparison. For PB-ALOHA, we use the estimated arrival rate $\hat{\lambda}_{t}=e^{-1}$, as suggested in [14]. $\mathrm{Q}^{+}$-Algorithm belongs to the class of multiplicative schemes which is first proposed by Hajek and van Loon [10]. In $\mathrm{Q}^{+}$. Algorithm, $\hat{N}_{t}$ is updated as follows:

$\hat{N}_{t+1}=\max \left\{1,\left[I\left(Z_{t}=0\right) / \zeta_{0}+I\left(Z_{t}=1\right)+\zeta_{c} I\left(Z_{t}=c\right)\right] \hat{N}_{t}\right\}$, where $\zeta_{0}=2^{0.25} \approx 1.1892$ and $\zeta_{c}=2^{0.35} \approx 1.2746$ are suggested in [12] for optimal performance.

Second, we describe the simulation scenarios. In order to gain more insights into the performance of access schemes with bursty traffic, we present simulation results for a single active stage and assume that the intervals between two consecutive active stages are sufficiently large so that all the devices can successfully access the BS before the next trigger. During the active stage, $N$ devices are triggered according to the beta distribution with parameters $\alpha=3, \beta=4$ [6], and time span
$T=50$ slots, which is the number of slots in one second when the PRACH period [3] is $20 \mathrm{~ms}$. It is assumed in 3GPP that 30,000 devices can be triggered in 10 s [6], thus we choose $N$ to be 100 to 3,000 per second.

\section{B. Results}

In some event-driven M2M applications, response can be taken with partial messages from the detecting devices and not all devices need to report an event. Thus, both the distribution of access delay and average delay are evaluated to study the performance of the proposed scheme.

Fig. 2 shows the cumulative distribution function of access delay for different schemes. From this figure we can see that the performance of the proposed FASA scheme is close to the benchmark with perfect knowledge. For PB-ALOHA, it takes a long time to track the number of backlogged devices and few devices can access successfully during this period. For instance, the $10 \%$ delay, which is the access delay achieved by $10 \%$ of the active devices, is much larger than other schemes. For example, when $N=500$, the $10 \%$ delay of FASA is about 280 slots while it is 520 slots for PB-ALOHA. With multiplicative increment, the $\mathrm{Q}^{+}$-Algorithm can track the number of backlogs in a short time because of the exponential increment due to the consecutive collision slots. However, it takes longer for all the devices to access the channel under $\mathrm{Q}^{+}$-Algorithm than under FASA due to the large estimation fluctuation in $\mathrm{Q}^{+}$-Algorithm.

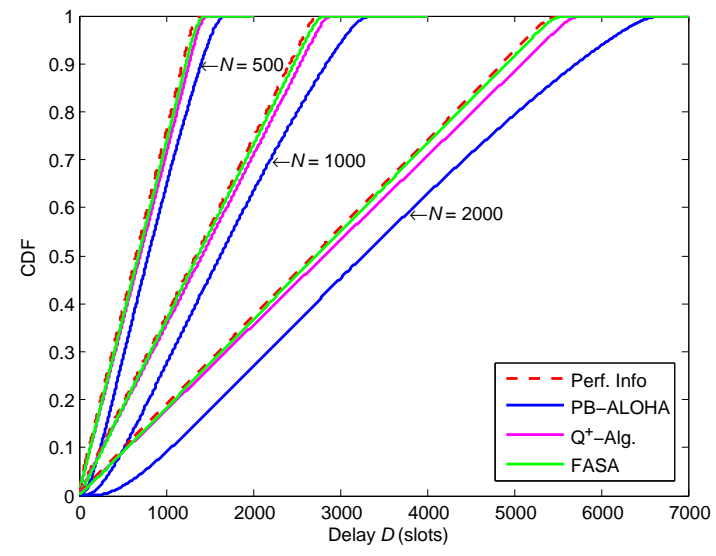

Fig. 2. Cumulative distribution function of access delay

Fig. 3 compares the average delay of the access schemes. The top figure indicates that the average delay increases almost linearly as the number of active devices increases. To quantify the divergence from the theoretical optimum performance, we define the normalized divergence as $e(D)=\frac{D-D^{*}}{D^{*}} \times 100 \%$, where $D$ is the average delay of a particular scheme and $D^{*}$ is the theoretical optimum delay with perfect information. The bottom part of Fig. 3 shows the divergence of PB-ALOHA, $\mathrm{Q}^{+}$-Algorithm, and FASA. The delay of PB-ALOHA scheme is larger than the optimum value by about $22 \%$. The divergences of $\mathrm{Q}^{+}$-Algorithm and FASA are both much less than that of PB-ALOHA. As the number of the active devices increases, the effect of estimation fluctuation becomes small and the performance gets close to the optimum value. For large $N$, the divergence is about $5 \%$ for $\mathrm{Q}^{+}$-Algorithm and $2 \%$ for FASA, which indicates that FASA scheme performs slightly better than $\mathrm{Q}^{+}$-Algorithm. 
As shown in Figs. 2 and 3, for a single active stage, the performance of FASA and $\mathrm{Q}^{+}$-Algorithm is close, with the proposed FASA scheme performing slightly better. However, when considering the long term performance, as discussed in [10], for given $\zeta_{0}$ and $\zeta_{1}$, the estimated value $\hat{N}_{t}$ continues to fluctuate when it gets close to $N_{t}$ and the stable throughput is less than some maximum value $\Theta_{\max }<e^{-1}$. We verify this property through simulations for Poisson arrival process with mean $\lambda$. The results show that, for PB-ALOHA and FASA, the number of backlogged devices is finite though the average value becomes large as $\lambda$ becomes close to $e^{-1}$. For $\mathrm{Q}^{+}$. Algorithm with the given parameter values, however, when $\lambda$ is larger than about 0.36 , the number of backlogged devices grows unbounded, indicating that the algorithm is unstable when $\lambda>0.36$.
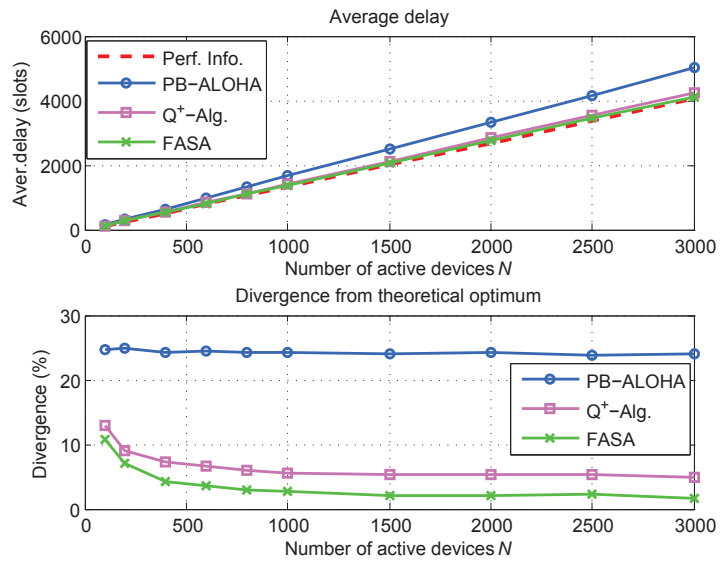

Fig. 3. Average delays for different schemes

\section{CONCLUSION AND Future WORK}

This paper proposes a fast adaptive S-ALOHA scheme, called FASA, for event-driven M2M communications. An approximate drift analysis and simulation results show that using FASA, a BS can track the number of backlogged devices more quickly. That is a main advantage compared to fixed step-size additive schemes, e.g., PB-ALOHA. Compared to multiplicative schemes, simulation results show that the proposed FASA scheme has better stability performance under heavy load in addition to slightly better delay performance. Currently, the analysis presented in this paper is based on the approximation with fixed number of backlogged devices. A rigorous analysis about the properties of the proposed scheme with general arrival traffic, e.g., mixed bursty and Poisson traffic, is left as our future work.

\section{ACKNOWLEDGEMENT}

This work was supported by the National Basic Research Program of China (973 Program, Grant No. 2010CB731803).

\section{APPENDIX: ProOF OF PROPOSITION 1}

The proposition can be proved by calculating the derivative of $\Delta_{F A S A}(\rho)$.

For a given value of $\nu$, let

$$
g_{0}(\rho)=\left|q_{0}(\rho) E\left[\Delta_{0}(\rho)\right]\right|=q_{0}(\rho)\left[1+h_{0}(\nu) M\left(\nu, q_{0}(\rho)\right)\right],
$$

and

$g_{c}(\rho)=\left|q_{c}(\rho) E\left[\Delta_{c}(\rho)\right]\right|=q_{c}(\rho)\left[\frac{1}{e-2}+h_{c}(\nu) M\left(\nu, q_{c}(\rho)\right)\right]$.

Then $g_{0}(1)=g_{c}(1)=e^{-1}+\eta$ and $\Delta_{F A S A}(1)=-g_{0}(1)+$ $g_{c}(1)=0$. Next, we claim that, for given $\nu>0, M(\nu, q)=$ $\sum_{k=1}^{\infty} k^{\nu} q^{k-1}(1-q)$ is an increasing function of $q(0<q<1)$, because

$$
\begin{aligned}
\frac{\partial M}{\partial q}= & \sum_{k=1}^{\infty} k^{\nu} q^{k-2}[k(1-q)-1] \\
= & \sum_{k=1}^{k^{*}} k^{\nu} q^{k-2}[k(1-q)-1] \\
& +\sum_{k=k^{*}+1}^{\infty} k^{\nu} q^{k-2}[k(1-q)-1] \\
> & \left(k^{*}\right)^{\nu} \sum_{k=1}^{\infty} q^{k-2}[k(1-q)-1]=0,
\end{aligned}
$$

where $k^{*}=\left\lfloor\frac{1}{1-q}\right\rfloor$ is the largest integer not greater than $\frac{1}{1-q}$, and thus $k^{\nu} \leq\left(k^{*}\right)^{\nu}$ if $1 \leq k \leq k^{*}$ and $k^{\nu}>\left(k^{*}\right)^{\nu}$ if $k>k^{*}$. In addition, the idle probability $q_{0}(\rho)=e^{-\rho}$ is nonnegative and strictly decreasing in $\rho$. Hence, $M\left(\nu, q_{0}(\rho)\right)$ is strictly decreasing in $\rho$ and $g_{0}(\rho)$ is a strictly decreasing function of $\rho$. On the other hand, since $q_{c}(\rho)=1-e^{-\rho}-\rho e^{-\rho}$ is nonnegative and strictly increasing in $\rho$, we can show similarly that $g_{c}(\rho)$ is a strictly increasing function of $\rho$. Thus, $\Delta_{F A S A}(\rho)=-g_{0}(\rho)+g_{c}(\rho)$ is a strictly increasing function of $\rho$. Consequently, $\Delta_{F A S A}(\rho)<\Delta_{F A S A}(1)=0$ when $0<\rho<1$ and $\Delta_{F A S A}(\rho)>\Delta_{F A S A}(1)=0$ when $\rho>1$.

\section{REFERENCES}

[1] 3GPP TS 22.368 V11.0.2, "Service requirements for machine-type communications," Jun. 2011.

[2] Cisco, "Cisco visual networking index: Global mobile data traffic forecast update, 2010-2015," Feb. 2011. [Online]. Available: http://www.cisco.com/en/US/netsol/ns827/networking_solutions_white_pa

[3] P. Bertrand and J. Jiang, LTE - The UMTS Long Term Evolution: From Theory to Practice. John Wiley \& Sons Ltd., 2009, ch. 19.

[4] S.-Y. Lien and K.-C. Chen, "Massive access management for QoS guarantees in 3GPP Machine-to-Machine communications," IEEE Communications Letters, vol. 15, no. 3, pp. 311 - 313, Mar 2011.

[5] R. Y. Kim, "Efficient wireless communications schemes for machine to machine communications," Communications in Computer and Information Science, vol. 181, no. 3, pp. 313 - 323, 2011.

[6] ZTE, "R2-104662: MTC simulation results with specific solutions," in 3GPP TSG RAN WG2 Meeting \#71, Aug. 2010.

[7] CATT, "R2-100182: Access control of MTC devices," in 3GPP TSG RAN WG2 Meeting \#68bis, Jan. 2010.

[8] G. Wang, X. Zhong, S. Mei, and J. Wang, "An adaptive medium access control mechanism for cellular based Machine to Machine (M2M) communication," in Proc. of IEEE ICWITS 2010, 2010.

[9] R. L. Rivest, "Network control by Bayesian broadcast," IEEE Tran. on Information Theory, vol. 33, no. 3, pp. 323 - 328, May 1987.

[10] B. Hajek and T. van Loon, "Decentralized dynamic control of a multiaccess broadcast channel," IEEE Tran. on Automatic Control, vol. 27, no. 3, pp. $559-569$, Jun. 1982.

[11] ISO/IEC, "ISO/IEC 18000-6:2010 information technology - radio frequency identification for item management - part 6: Parameters for air interface communications at $860 \mathrm{MHz}$ to $960 \mathrm{MHz}, 2010$.

[12] D. Lee, K. Kim, and W. Lee, " $\mathrm{Q}^{+}$-algorithm: An enhanced RFID tag collision arbitration algorithm," Lecture Notes in Computer Science, Ubiquitous Intelligence and Computing, vol. 4611, no. 31, pp. 23 - 32, 2007.

[13] F. P. Kelly, "Stochastic models of communication systems," Journal of the Royal Stastical Society (Series B), vol. 47, no. 3, pp. 379-395, 1985.

[14] J. N. Tsitsiklis, "Analysis of a multiaccess control scheme," IEEE Tran. on Automatic Control, vol. 32, no. 11, pp. 1017 - 1020, Nov. 1987. 